\title{
THE RATAN-600 - VLA - 6M RUSSIAN TELESCOPE: EARLY UNIVERSE PROJECT
}

\author{
Y.N. PARIJSKIJ ${ }^{1}$, N.S.SOBOLEVA ${ }^{1}$, W.M. GOSS ${ }^{2}$, A.I. KOPYLOV ${ }^{1}$, \\ O.V. VERKHODANOV ${ }^{1}$, A.V. TEMIROVA ${ }^{1}$ AND O.P. ZHELENKOVA ${ }^{1}$ \\ 1 Special Astrophysical Observatory of the Russian Academy \\ of Sci - 357147 Nizhnij Arkhyz Karachaj-Cherkessia, RUSSIA \\ ${ }^{2}$ National Radio Astronomocal Observatory - Edgemont Road, \\ Charlottesville VA 22903 U.S.A.
}

We present the preliminary results of the "BIG TRIO" project of penetration into the "Dark Age" of the Universe, between the recombination epoch and the epoch of the first QSO using RATAN-600 "Cold Experiment" deep (few mJy) multi-frequency strip survey (Parijskij, Korolkov, 1986; Parijskij et.al,1991,1992). Our general approach is close to the classical SS FRII RG selection rules of very distant galaxies with old stellar population (McCarthy, 1993) with small improvements. Details may be found in (Kopylov et al, 1995). Mean estimated $z$ of our SS FRII RG's is about $1.5,20 \%$ of all objects have $z$ larger than 3 . Before the direct spectroscopy of all SS FRII objects we have estimated their redshifts by different methods: using the updated Hubble diagram, $\theta-z$ and flux density$z$ relations and now begun to use multi-color measuring all our objects to estimate "color $z$ " and a stellar age of the parent galaxies using available models of stellar population evolution in such objects (gE class). The first subgroup of 16 objects (not the weakest ones) measured in BVRI gave us the following preliminary results using Bruzual-Charlot model (Bruzual et al., 1993). Looking at this Table, we can make the following statements: mean color $z$ occurred to be very close (within 15\%) to the photometric $z$, but individual redshifts may differ. A mean age of the stellar population is about $1 \mathrm{Gyr}$, and, at least in some objects, star formation began when the Universe was only $1 \mathrm{Gyr}$ old $\left(\Omega_{0}=1, H_{0}=50\right)$, that is older than the most distant QSOs. One object, RC0934+0505, happened to be the oldest object found up to now. We estimate, that there are about 10000 objects 
TABLE 1. STARS FORMATION EPOCH FROM "BIG TRIO" PROJECT

$\begin{array}{lcccccccc}\text { N } & \text { RC-name } & \text { B-V } & \text { V-R } & \text { R-I } & \text { R } & \text { Z-rg } & \text { Star age, Gyr } & \text { Z-star formation } \\ 1 & 0837+0446 & 0.01 & 0.79 & -0.04 & 22.17 & 1.5 & 0.33 & 1.7 \\ 2 & 0908+0451 & 0.95 & 0.90 & 0.81 & 19.96 & 0.5 & 1.1 & 0.7 \\ 3 & 0934+0505 & 0.87 & -0.13 & 1.38 & 24.07 & 3.4 & 1.1 & 9.5 \\ 4 & 1031+0443 & 1.17 & 0.72 & 1.27 & 22.18 & 1.0 & 1.1 & 1.4 \\ 5 & 1152+0449 & 0.22 & 1.28 & 1.41 & 22.45 & 1.0 & 1.1 & 1.4 \\ 6 & 1155+0444 & 1.55 & 0.94 & 0.71 & 18.95 & 0.5 & 1.1 & 0.7 \\ 7 & 1219+0446 & 0.61 & 0.84 & 0.30 & 22.25 & 2.3 & 1.1 & 4.1 \\ 8 & 1333+0452 & 0.46 & 0.89 & 1.12 & 23.64 & 1.1 & 0.9 & 1.4 \\ 9 & 1357+0453 & 1.16 & 0.76 & 1.01 & 21.17 & 0.5 & 1.1 & 0.7 \\ 10 & 1436+0501 & 0.07 & 0.50 & 0.71 & 23.47 & 2.0 & 0.6 & 2.6 \\ 11 & 1510+0438 & 1.30 & 1.19 & 1.35 & 22.69 & 0.6 & 1.7 & 0.9 \\ 12 & 1626+0448 & -0.69 & 0.37 & 0.14 & 22.88 & 2.6 & 0.35 & 3.1 \\ 13 & 1646+0501 & 1.66 & 1.53 & 1.28 & 21.21 & 0.7 & 1.7 & 1.1 \\ 14 & 1703+0502 & 0.96 & 0.35 & 0.96 & 23.47 & 3.4 & 0.6 & 5.2 \\ 15 & 1740+0502 & 1.83 & 0.55 & 0.64 & 22.63 & 0.6 & 0.9 & 0.8 \\ 16 & 2013+0508 & 0.76 & 0.29 & 0.23 & 21.12 & 2.9 & 0.4 & 3.6\end{array}$

with such an age on the sky which can be easily selected (especially with new generation of radio and optical catalogs now in preparation) and fully studied spectroscopically even by present day facilities. The only evolution effect we have noticed is that more distant objects have smaller ages of stellar populations. There is weak $z$-dependence of the ratio of radio to optical luminosities in the objects of our list, which disappears when we take into account the K- correction to the SED in $\mathrm{gE}$.

This work was done with partial support by ISF Grant 96300 , Russian "Cosmion" and RFFI grants 93-02-1738, 95-02-03783.

\section{References}

Bruzual G, Charlot, S. (1993) Ap.J., 405, 538.

McCarthy, P. (1993) Ann.Rev. of $A \& A$ \&., 31, 639.

Kopylov A., Goss W., Parijskij Y., Soboleva N., Zhelenkova O., Temirova A., Vitkovskij Val., Naugolnaya M., Verkhodanov O. (1995) it A.J.,Russian, 72, 437.

Parijskij, Y., Korolkov, D. (1986) Ap\& Space Phys.Rev., 5,40.

Parijskij, Y., Bursov, N., Lipovka, N., Temirova, A. (1991) A \&A Suppl.Ser., 87, 1.

Parijskij, Y., Bursov, N., Lipovka, N., Soboleva, N., Temirova, A., Chepurnov, A. (1992) $A \mathcal{B} A$ Suppl.Ser., 96, 583. 